Introduction: Osteosarcoma is the most common primary malignant bone tumor in adults and is usually located in long bones. Standard treatment consists of perioperative chemotherapy and radical surgical resection. In the case of the extremity location, the gold standard is limb-sparing surgery (LSS) using a variety of reconstructive techniques.

Aim of the study: To assess long-term results of adults patients treated for limb osteosarcoma in our referral center depending on the method of surgical treatment.

Material and methods: In our study, we analyzed 175 adult patients with localized disease (American Joint Committee on Cancer [AJCC] stage I-III) treated for extremity osteosarcoma at our institution between 2000 and 2017. The median observation time was 41 months (3-225 months). 111 patients were treated with LSS (80 patients had tumor resection followed by endoprosthetic reconstruction, 31 patients had local resection without reconstruction) and $64 \mathrm{pa}$ tients underwent amputation.

Results: 5-year overall survival (OS) and progression-free survival (PFS) in the study group were $62 \%$ and $52 \%$ and the life expectancy was on average 136 months. In the group of patients treated with LSS, 5-year OS and PFS were $66 \%$ and $59 \%$, respectively, and life expectancy was 147 months, while in the group of patients undergoing amputation 5 -year OS, PFS and life expectancy were $55 \%, 42 \%$ and 117 months.

Conclusions: The best results in the treatment of extremity osteosarcoma were achieved in a group of patients without distant metastases at the time of diagnosis, treated with perioperative chemotherapy and radical resection followed by endoprosthetic reconstruction.

Key words: osteosarcoma, combined treatment, limb reconstruction, amputation.

Contemp Oncol (Pozn) 2019; 23 (4): 234-238 DOI: https://doi.org/10.5114/wo.2019.89782

\section{Long-term follow-up in adults with extremity osteosarcoma: comparison of different surgical procedures - single-center experience}

\author{
Tomasz Goryń, Bartłomiej Szostakowski, Andrzej Pieńkowski, \\ Marcin Zdzienicki, Iwona Ługowska, Piotr Rutkowski
}

Department of Soft Tissue/Bone Sarcoma and Melanoma, Memorial Cancer Center and Institute of Oncology, Warsaw, Poland

\section{Introduction}

Osteosarcoma is the most commonly diagnosed primary malignant bone tumor, and it peaks in puberty and the fifth decade of life. Incidence of osteosarcoma in the population is 0.2 per 100,000 , which is less than $1 \%$ of solid tumors [1, 2]. The most common locations of osteosarcoma include long bones forming the knee joint, then the proximal femur and the proximal humerus. The tumor is less frequently located in the pelvis, flat bones, vertebrae and other short bones. The best treatment results are obtained using combined therapy that includes perioperative chemotherapy followed by radical surgical resection.

Long-term 5-year or 10-year overall survival (OS) in patients with osteosarcoma varies from $50 \%$ to $80 \%$. However, they are strictly dependent on many prognostic factors, which include the stage of disease (distant metastases) at the time of diagnosis, presence of pathological fracture, histopathological grade (G3), the use of appropriate multidisciplinary treatment, including the possibility of radical resection surgery, and the age of the patient. In children and adolescents, long-term treatment results are relatively good, and 5-year OS is around $70-80 \%$, while in young adults, especially in patients over 40, they are at the level of 40-50\% [3-5].

Due to the rarity of the disease, diagnosis and treatment of patients with osteosarcoma is a great challenge, and hence should be carried out in cooperation with reference centers, in particular its surgical treatment. At present, limb-sparing surgery (LSS) can be performed in the majority of patients with limb osteosarcoma. Various reconstructive techniques are used to restore the functional limb after resection of bones and joints.

The most frequently used are reconstructions with endoprosthetic replacements, arthrodesis implants, autografts, allografts and custom-made implants. Despite the increased risk of local recurrence, this is currently considered as the optimal treatment procedures. Published research shows that patients undergoing such treatment are characterized by a longer OS rate as compared with patients after amputation, not to mention physical disability and its psychological effects that occur in patients undergoing such a mutilating procedure $[6,7]$.

Amputation is necessary only in about $10-35 \%$ of patients with extensive involvement of surrounding soft tissue, vessels or nerves, in poor general condition or after improper, earlier surgical treatment. Patients with non-radical resection (R1) have a significantly worse prognosis than patients with radical resection (RO), while in patients with non-operable changes the prognosis is very poor and patients usually die due to tumor progression despite intensive chemotherapy. 
The aim of the study was to assess long-term results of adults patients treated for limb osteosarcoma in our referral center depending on the method of surgical treatment.

\section{Material and methods}

Between 2000 and 2017, 206 adult patients with extremity osteosarcoma were surgically treated at our institution. Thirty one patients with stage IV disease according to American Joint Committee on Cancer (AJCC) were excluded from the analysis (metastasis at the time of diagnosis). One hundred seventy-five adult patients without distant metastases who were treated for limb osteosarcoma at our department in the period 2000-2017 were included in this retrospective analysis.

All patients with a confirmed diagnosis were treated with 3-4 courses of preoperative chemotherapy based on cisplatin and doxorubicin. Then, 3-4 weeks after completing their last preoperative chemotherapy course, the sarcoma resection was performed either by amputation or with a LSS.

After surgery, patients received a further 3-6 chemotherapy courses according to the preoperative schedule. In case when the diagnosis of osteosarcoma was established after the primary "whoops" (unplanned operation without diagnosis) operation, patients received all (6-9) courses of adjuvant chemotherapy according to the same pattern.

All patients remained under the care of the department's outpatient clinic and mortality data were obtained from the National Cancer Registry. The characteristics of the analyzed cohort of patients are presented in Table 1.

\section{Statistical analysis}

All statistical analyses were performed using the SPSS statistical program. For the survival analysis, the Kaplan-Meier estimator was used with the log-rank tests for bivariate comparisons. The objective was to assess the relationship between clinical parameters and OS, local relapse-free survival (LRFS) and progression-free survival (PFS) of non-metastatic osteosarcoma patients treated surgically and with chemotherapy. OS time was calculated from the date of the second-opinion, pathological report to the date of the most recent follow-up or death. Dates of all deaths were confirmed in the National Death Registry. PFS time was calculated from the date of the second-opinion, biopsy pathological report to the date of the most recent follow-up, or progression or death due to the disease. In multivariate analysis of the factors associated with OS, PFS and LRFS we used Cox proportional hazards models. The differences were considered statistically significant if the $p$-values were $<0.05$.

\section{Results}

The mean age in the whole group of patients was 35 years (18-81 years). Men in the study group accounted for $61 \%$ (106 patients).

Extremity osteosarcoma occurred in the lower limb in 140 patients, while it was located in the upper limb in 35 patients.

In the lower limb, the tumor was located in the femur in 92 patients, including the distal metaphysis in 69 patients, in the proximal metaphysis in 12 patients and the diaphysis in 11 patients. In 34 patients, the tumor was found in the tibia, including the proximal part in 26 patients. In the fibula, sarcoma occurred in 11 patients, while in the bones of the foot it occurred in 3 patients. In the upper limb, the tumor was located in the proximal part of the humerus in 25 patients, and in the remaining bones of the upper limb in 10 patients. The treatment modalities in the analyzed group are shown in Figure 1 and Table 2.

The median follow-up in the study group was 48 months. The 5 -year OS for the study group was $62 \%$, while the mean length of OS in the study group was 136 months (3-225 months).

The group of patients who underwent the LSS was compared with a group of patients who underwent amputation. 5-year survival, disease-free survival, local recurrence, metastatic disease, presence of adverse prognostic factors in the examined groups, and radical resection (RO) were assessed.

In the group of patients undergoing a LSS, long-term OS results were more favorable in comparison to the group of patients who underwent amputation and were $65 \%$ vs. $55 \%$. The average life expectancy in the group of patients who underwent LSS was 146 months and was 29 months longer than in the group of patients undergoing amputation. In the case of PFS assessment, the difference in favor of the group treated in a saving way was statistically significant ( $p=0.019$ ) (Fig. 2).

Table 1. Patients' characteristics and 5-year overall survival and progression-free survival depending on clinical parameters

\begin{tabular}{|c|c|c|c|c|c|}
\hline Osteosarcoma patients, MO & $n$ & 5-year OS (\%) & $p$-value & PFS (\%) & $p$-value \\
\hline Female & 68 & 63 & 0.732 & 54 & 0.702 \\
\hline Male & 107 & 61 & & 51 & \\
\hline Age $<25$ years & 62 & 64 & 0.422 & 59 & 0.072 \\
\hline Age $>25$ years & 113 & 61 & & 48 & \\
\hline Pathological fracture present & 29 & 67 & 0.682 & 46 & 0.205 \\
\hline Pathological fracture absent & 146 & 61 & & 52 & \\
\hline Limb-sparing surgery (LSS) & 111 & 66 & 0.88 & 57 & 0.205 \\
\hline Amputation & 64 & 55 & & 43 & \\
\hline RO resection & 142 & 64 & 0.1 & 58 & 0.001 \\
\hline R1 resection & 33 & 53 & & 23 & \\
\hline
\end{tabular}

OS - overall survival, PFS - progression-free survival 


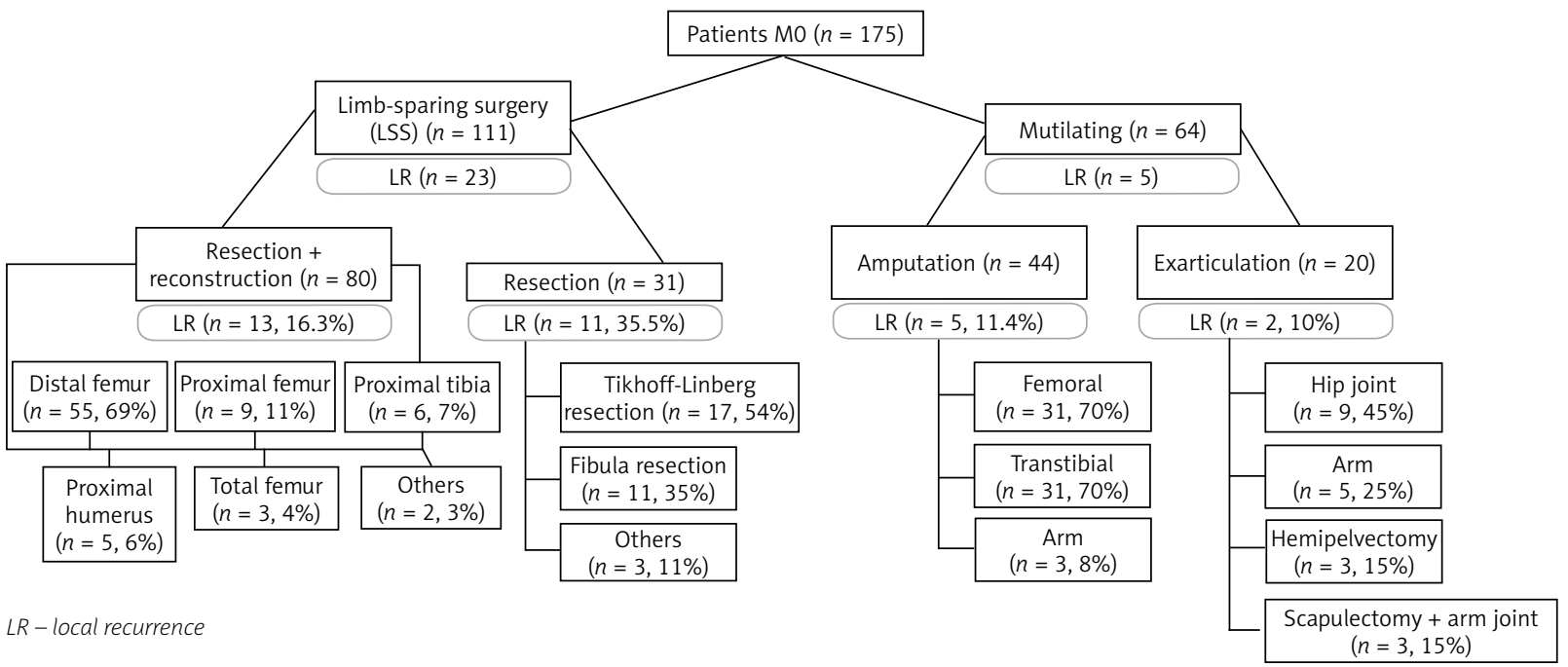

Fig. 1. Treatment modalities depending on the location of the sarcoma

Table 2. Treatment methods depending on the location of osteosarcoma

\begin{tabular}{|c|c|c|c|c|c|c|c|c|}
\hline Method & $\begin{array}{l}\text { Distal } \\
\text { femur }\end{array}$ & $\begin{array}{l}\text { Proximal } \\
\text { femur }\end{array}$ & $\begin{array}{c}\text { Femur } \\
\text { shaft }\end{array}$ & $\begin{array}{l}\text { Proximal } \\
\text { tibia }\end{array}$ & $\begin{array}{l}\text { Proximal } \\
\text { humerus }\end{array}$ & Fibula & Others & Total \\
\hline Reconstruction surgery & 55 & 9 & 3 & 6 & 5 & 0 & 2 & 80 \\
\hline PF & 2 & 2 & 2 & 0 & 0 & 0 & 0 & 6 \\
\hline Mutilating & 14 & 3 & 7 & 19 & 5 & 2 & 14 & 64 \\
\hline PF & 7 & 0 & 4 & 0 & 4 & 0 & 4 & 19 \\
\hline Resection & 1 & 0 & 0 & 1 & 17 & 9 & 3 & 31 \\
\hline PF & 0 & 0 & 0 & 1 & 3 & 0 & 3 & 7 \\
\hline
\end{tabular}

PF- pathological fracture

Figure 3 shows 5-year OS depending on the treatment method. The best results were achieved in the group of patients undergoing LSS surgery. The results obtained in the group of patients undergoing amputation or local resection without reconstruction were slightly worse. In contrast, the worst results were obtained in the group of patients who underwent disarticulation of the shoulder, hip joint or hemipelvectomy.

Histopathological resection RO was performed in 142 (81\%) patients. Compared to the group of 33 patients with

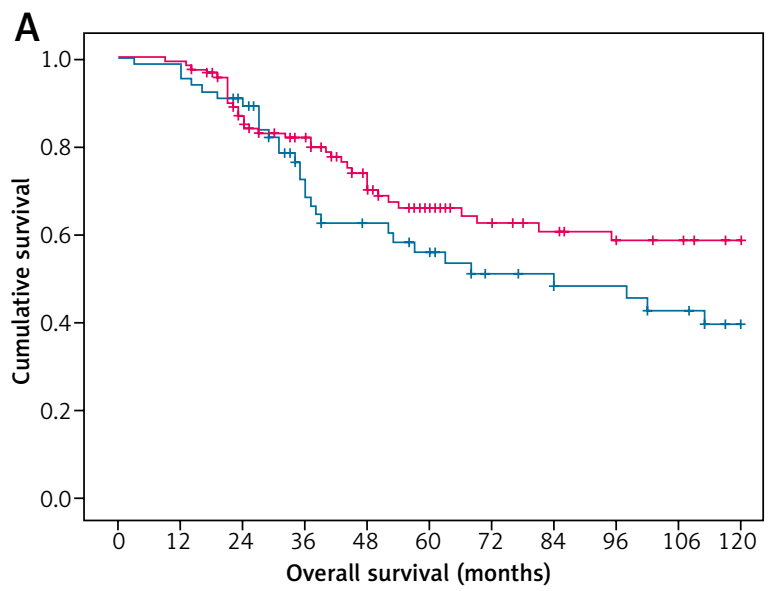

resection (R1), the patients with $\mathrm{RO}$ resection were characterized by better 5 -year OS and statistically significantly better PFS (Fig. 4).

One of the significant risk factors worsening the prognosis of patients treated for osteosarcoma tends to be the presence of a pathological fracture. In the group of analyzed patients, 29 (16\%) of them had a pathological fracture as the initial symptom. In these patients, the treatment of choice was amputation, which was performed in 19 patients (65\%), while LSS was performed in 10 patients. Our result showed

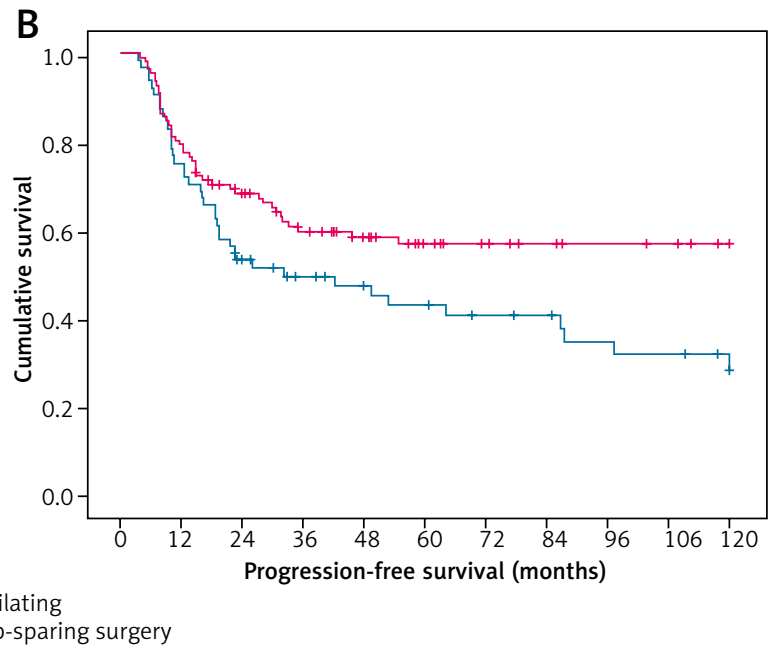

Fig. 2. 5-year overall survival and progression-free survival in the group of patients treated with limb-sparing surgery vs. amputation 
no difference in long-term survival (5-year OS) and 5-year PFS in patients with or without pathological fracture

In COX multivariate analysis, factor significantly influencing the 5-year OS in patients with localize osteosarcoma included non-radical resection $\mathrm{R} 1(p=0.035)$ and the possibility of performing LSS $(p=0.052)$. Other factors, such as age and gender of patients, and the presence of a pathological fracture, did not affect the prognosis.

\section{Discussion}

Our institution is a reference center for the treatment of adult osteosarcoma and soft tissue sarcoma, and for the past 25 years had conducted comprehensive treatment of patients with primary bone tumors, including osteosarcoma. Our department is a part of EURACAN reference center and one of a few centers dedicated to treatment of patients with bone sarcomas.

Initially, at our institution, LSS reconstructions for osteosarcoma were performed with custom made implants dedicated to particular patients. Since 2003, we have used modular systems by Modular Universal Tumor And Revision System (MUTARS).

The 5-year OS results of treatment of patients at our center are comparable to those presented by leading units in the world. The diagnostic and therapeutic procedures are identical to the European and American recommendations.

The results of 5-year OS with the localized stage are $61 \%$. This is a result similar to the data presented by other European centers. The results presented by O'Kane and Bacci on 5 -year survival are similar (OS 64\% and 67\%) even though the study group also included patients under 18 years of age, for which the results of treatment are more favorable.

In the work of the German group, in which the study group consisted of 1709 patients, OS was 48.9\% and was slightly worse than the survival results obtained in our group [3-6].

According to our observation, the occurrence of a pathological fracture with an appropriate treatment does not affect the patients' prognosis. Li in his work presented similar observations [7]. Ferguson presented the

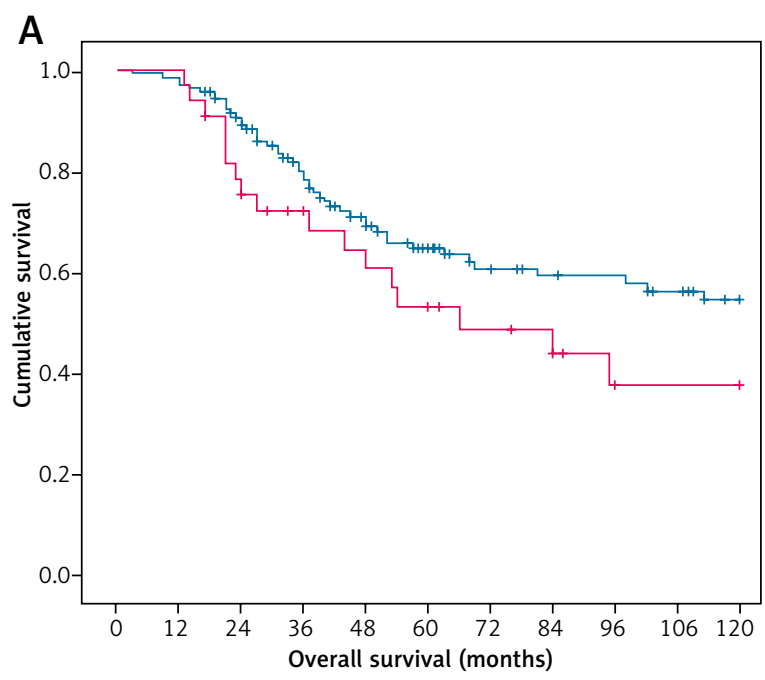

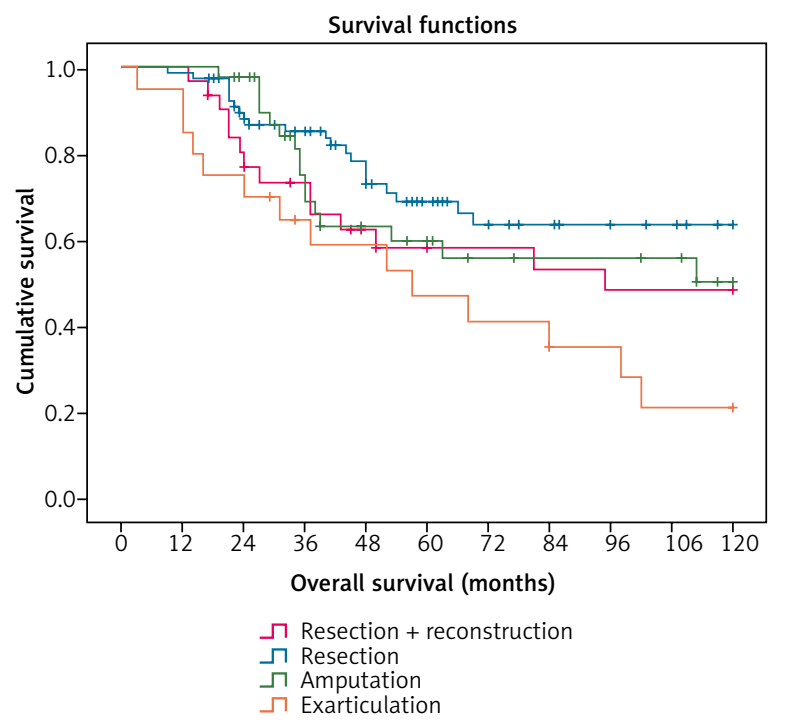

Fig. 3. 5-year overall survival depending on the type of surgical treatment

opposite results. In the group of patients analyzed by him, the presence of a pathological fracture resulted in a worse prognosis of patients undergoing treatment [8]. So far, it seems that in the case of the presence of a pathological fracture, the surgical treatment of choice is amputation [9]. In our study group, the majority of patients who had a pathological fracture underwent amputation. In our results, patients treated with LSS had a favorable long-term outcome with better quality of life.

Surgical treatment of the primary tumor includes LSS, resections without subsequent reconstruction and amputations. In our material, amputations account for as much as $36 \%$, which is a slightly higher result than in other European centers. In published papers, the percentage of amputations was between 10 and 30\%, but most of them include treatment of children, in whom amputation is performed much less frequently. In adults, more advanced disease at the time of diagnosis or the presence of a fracture causes an increased rate of mutilating treatment.

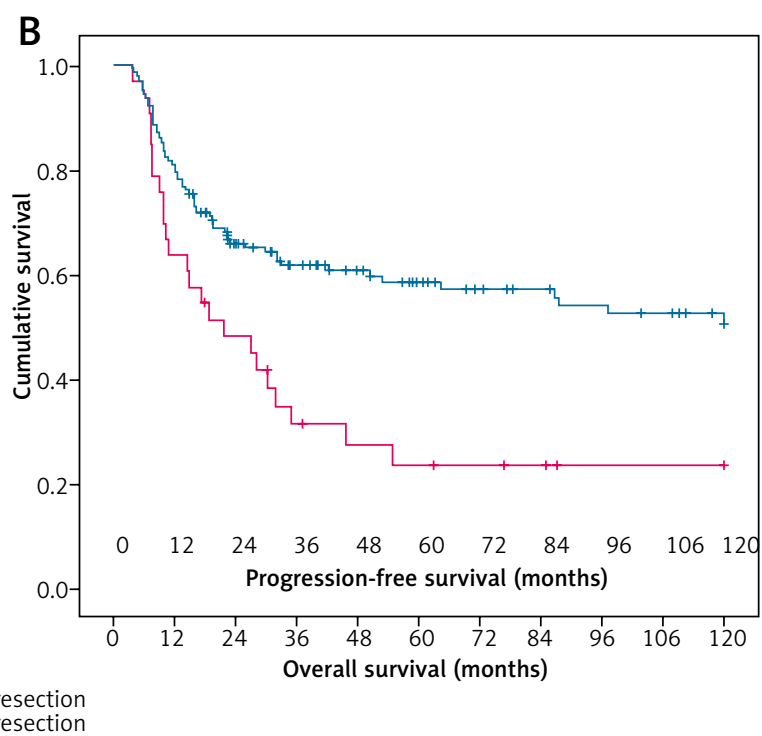

Fig. 4. 5-year overall survival in patients with RO vs. R1 resection 
In large two metaanalyses involving more than 1,300 patients, our observations were confirmed where also a worse prognosis was found in patients who underwent amputation [10-12]. However, He et al. presented different data in his meta-analysis, in which he did not find a statistically significant difference in the 5-year OS and the incidence of local recurrence in patients treated with a salvaging procedure and amputation [13]. However, as the authors themselves admit, the group assessed was characterized by considerable heterogeneity and a significant part of the studied group was a yellow race population. After exclusion from the group of the most heterogeneous groups of patients, the obtained results indicated more favorable results in the group subjected to LSS treatment.

The patients who underwent amputations usually had other adverse risk factors such as the occurrence of a pathological fracture, involvement of vessels and nerves, a larger size of the primary tumor, local recurrence after inappropriate surgical treatment in a non-specialized center or the worse general condition of the patients, and it could result in a worse treatment outcome.

In our study group, the prognosis was not affected by the age of the patient. This is different from the described works, in which there was a significant worsening of the prognosis in patients after 40 years of age. In our study group, these patients constituted a minority, and this fact may be the cause of the lack of statistical significance. Our group included only adults, which may also have affected this result.

As in other studies, non-radical resection negatively affects patients' prognosis regarding long-term survival and local recurrence.

\section{Conclusions}

Correctly planned and performed combination therapy in patients with osteosarcoma according to our observations is crucial for the further prognosis of patients and is the only factor that is modifiable and dependent on medical practice.

The most favorable treatment method in adult patients with extremity osteosarcoma is the use of multidisciplinary planned combined therapy that includes perioperative chemotherapy followed by radical LSS.

In order to improve the results of treatment, it is necessary to seek the right diagnosis and initiate proper treatment in the reference center as soon as possible. Correctly carried out diagnostics and treatment will allow most osteosarcoma patients to undergo successful treatment of the disease and return to normal functioning and limitation of their disability. Reducing the number of amputations associated with the significant progression of osteosarcoma at the time of diagnosis is the desired condition.

In order to provide the best standard of treatment for patients with rare cancers and the introduction of optimal, both economically and time wise, diagnostics of these cancers, a network of reference centers dedicated to the treatment of this type of cancer was established. In these centers, international scientific research and patients have access to the latest therapies, including experimental therapies.

\section{Acknowledgements}

We greatly appreciate all the nursing staff of the Department of Soft Tissue/Bone Sarcoma and Melanoma Institute - Oncology Center Warsaw Poland for helping to collect and record all data.

The authors declare no conflict of interest.

\section{References}

1. Rutkowski P, Świtaj T, Mazurkiewicz T, et al. Bone sarcomas. Oncol Clin Pract 2018; 14: 115-128.

2. Casali PG, Bielack S, Abecassis N, et al. Bone sarcomas: ESMOPaedCan-EURACAN Clinical Practice Guidelines for diagnosis, treatment and follow-up. Ann Oncol 2018; 29 (Suppl 4): 79-95.

3. Bacci G, Longhi A, Versari M, Mercuri M, Briccoli A, Picci P. Prognostic factors for osteosarcoma of the extremity treated with neoadjuvant chemotherapy: 15-year experience in 789 patients treated at a single institution. Cancer 2006; 106: 1154-1161.

4. Bielack SS, Kempf-Bielack B, Delling G, et al. Prognostic factors in high-grade osteosarcoma of the extremities or trunk: an analysis of 1,702 patients treated on neoadjuvant cooperative osteosarcoma study group protocols. J Clin Oncol 2002; 20: 776-790.

5. Briccoli A, Rocca M, Salone M, Guzzardella GA, Balladelli A, Bacci G. High grade osteosarcoma of the extremities metastatic to the lung: long-term results in 323 patients treated combining surgery and chemotherapy, 1985-2005. Surg Oncol 2010; 19: 193-199.

6. O'Kane GM, Cadoo KA, Walsh EM, et al. Perioperative chemotherapy in the treatment of osteosarcoma: a 26-year single institution review. Clin Sarcoma Res 2015; 5: 17.

7. Li X, Zhang Y, Wan S, et al. A comparative study between limb-salvage and amputation for treating osteosarcoma. J Bone Oncol 2016; 5: 15-21

8. Ferguson PC, McLaughlin CE, Griffin AM, Bell RS, Deheshi BM, Wunder JS. Clinical and functional outcomes of patients with a pathologic fracture in high-grade osteosarcoma. J Surg Oncol 2010; 102: 120-124.

9. Scully SP, Ghert MA, Zurakowski D, Thompson RC, Gebhardt MC. Pathologic fracture in osteosarcoma: prognostic importance and treatment implications. J Bone Joint Surg Am 2002; 84: 49-57.

10. Han G, Bi WZ, Xu M, Jia JP, Wang Y. Amputation Versus Limb-Salvage Surgery in Patients with Osteosarcoma: A Meta-analysis. World J Surg 2016; 40: 2016-2027.

11. Reddy KI, Wafa H, Gaston CL, Grimer RJ, Abudu AT, Jeys LM, Carter SR, Tillman RM. Does amputation offer any survival benefit over limb salvage in osteosarcoma patients with poor chemonecrosis and close margins? Bone Joint J 2015; 97-B: 115-120.

12. Jiang F, Shi Y, Li GJ, Zhou F. A meta-analysis of limb-savage versus amputation in the treatment of patients with Enneking II pathologic fracture osteosarcoma. Indian J Cancer 2015; 51 (Suppl 2): 21-24.

13. He X, Gao Z, Xu H, Zhang Z, Fu P. A meta-analysis of randomized control trials of surgical methods with osteosarcoma outcomes. J Orthop Surg Res 2017; 12: 5.

\section{Address for correspondence}

\section{Tomasz Goryń}

Department of Soft Tissue/Bone Sarcoma and Melanoma

Memorial Cancer Center and Institute of Oncology

5 W.K. Roentgena St.

02-781 Warsaw, Poland

e-mail: tomasz.goryn@coi.pl

Submitted: 1.10 .2019

Accepted: 25.10.2019 